\title{
Epidemiology, species distribution, and outcome of nosocomial Candida spp. bloodstream infection in Shanghai - A retrospective of 11-year clinical analysis in a shanghai tertiary hospital
}

\author{
Yan-Jun ZHENG \\ Shanghai Jiao Tong University Medical School Affiliated Ruijin Hospital \\ Ting XIE \\ Suining Central Hospital \\ Lin WU \\ Shanghai Jiao Tong University Medical School Affiliated Ruijin Hospital \\ Xiao-Ying LIU \\ Shanghai Jiao Tong University Medical School Affiliated Ruijin Hospital \\ Ling ZHU \\ ruijin north hospital \\ Ying CHEN \\ Shanghai Jiao Tong University Medical School Affiliated Ruijin Hospital

\section{En-Qiang MAO} \\ Shanghai Jiao Tong University Medical School Affiliated Ruijin Hospital \\ Li-Zhong HAN \\ Shanghai Jiao Tong University Medical School Affiliated Ruijin Hospital \\ Er-Zhen CHEN \\ Shanghai Jiao Tong University Medical School Affiliated Ruijin Hospital \\ Zhi-Tao YANG ( $\square$ yangzhitao@hotmail.fr) \\ Shanghai Jiao Tong University Medical School Affiliated Ruijin Hospital https://orcid.org/0000-0002-6289-6493
}

\section{Research}

Keywords: Candida spp., Bloodstream infection, Epidemiology, Species distribution, Antifungal therapy, Susceptibility, Early treatment, Survival

Posted Date: August 31st, 2020

DOI: https://doi.org/10.21203/rs.3.rs-63464/v1

License: @ (1) This work is licensed under a Creative Commons Attribution 4.0 International License. Read Full License 


\section{Abstract \\ Background:}

Candida spp. are important opportunist pathogens causing bloodstream infections (BSIs). The present study aims to describe the current epidemiology of Candida BSI in a large Shanghai Tertiary-care Hospital from 2008 to 2018 and to identify the risk factors and the impact of antifungal therapy on clinical outcomes.

\section{Methods:}

From January 2008 to December 2018, all consecutive patients who developed Candida BSI at Ruijin Hospital were enrolled. Underlying diseases, clinical severity, species distribution, antifungal therapy, and its impact on the outcome were analyzed.

\section{Results:}

The incidence of nosocomial Candida BSI was 0.39 episodes/1000 hospitalized patients, and the overall 28-day mortality rate was $28.5 \%$. Among the 393 cases of Candida BSI, 299 cases (76.1\%) received antifungal therapy. 247 received early appropriate antifungal therapy (an appropriate drug with adequate dosage was started before the subsequent in vitro susceptibility results), and 52 received target antifungal therapy (appropriate target treatment started after susceptibility results, regardless of whether the inappropriate antifungal treatment has been initialed or not). The 28-day mortality rate was significantly lower in those who received early appropriate antifungal therapy or target antifungal therapy compared with those who did not receive antifungal therapy $(25.5 \%$ and $23.1 \%$ vs. $39.4 \%, P=0.012$ and $P=0.046)$. In multivariate Cox regression analysis, age, chronic renal failure, mechanical ventilation, neutropenia were independent risk factors for the 28day mortality rate, while antifungal therapy was a protective factor for short-term survival rate.

\section{Conclusions:}

The epidemiology of Candida BSI in Shanghai differed from that observed in Western countries. Antifungal therapy did influence the shortterm survival, while there was no significant difference between the mortality for those who received early appropriate antifungal therapy and for those who received target antifungal therapy.

\section{Background}

The incidence of invasive fungal infection has increased over time, especially for Candida bloodstream infections (BSIs), which was associated with considerable excess mortality and costs. In the past two decades, the incidence of fungal infection has increased from 0.1 episodes/1000 admissions to 0.3-0.6 episodes/1000 admissions in China, North America, and some European countries[1-4]. The mortality rate is ranging from $35 \%-53 \%$ [5-8]. The optimal management of Candida BSIs included early awareness of patients at risk, control of the infection source, and timely administration of appropriate antifungal agents. Consequently, antifungal agents have been widely used as empirical therapy. While the overuse of antifungal agents resulted in increased costs, toxicity, ecological selection pressure for antifungal resistance and adverse drug interactions, etc. Several studies showed that delayed antifungal therapy (more than $48 \mathrm{~h}$ from onset) was associated with higher mortality[9], while others have yielded conflicting results[10-12].

In this study, we retrospectively analyzed all Candida bloodstream infection patients from 2008 to 2018 in our hospital, aiming to describe their clinical characteristics, species distribution, antifungal therapy and to identify its risk factors for 28-day mortality.

\section{Methods}

\section{Study Setting and population}

A retrospective analysis of consecutive Candida bloodstream episodes in adults collected from the microbiology database of a 1900-bed teaching hospital in Shanghai was performed over 11 years (from $1^{\text {st }}$ January 2008 to $31^{\text {st }}$ December 2018).

Demographics, underlying diseases, co-morbidities, the severity of clinical features, Candida species distribution, and early appropriate or target treatment were compared among the patients with Candida BSI. The initial and target antifungal agent use was also collected. 
A case of Candida BSI was defined as a patient with at least one blood culture positive for a Candida. [13]. Neutropenia was defined as $<500 / \mathrm{mm}^{3}$ absolute neutrophil count. Prior corticosteroid was defined as receiving $>1 \mathrm{mg} / \mathrm{kg} / \mathrm{d}$ prednisone for more than one week or equivalent before Candida BSI onset.

\section{Laboratory Methods}

Isolates were detected from blood cultures using the BACTEC ${ }^{T M}$ FX system (Becton Dickinson, Inc., Sparks, MD, USA). Flucytosine, amphotericin B, fluconazole, voriconazole, and itraconazole susceptibility testings were performed using the ATBß FUNGUS 3 system (BioMérieux, France), which was wildly used in China[14], providing susceptibility to antifungals results markedly concordant with those obtained using CLSI and EUCAST methodologies[15].

Early appropriate antifungal treatment was considered when the appropriate drug with adequate dosage was started before the subsequent in vitro susceptibility results. Adequate dosage of the antifungal agent was defined according to IDSA 2009 guidelines or 2016 IDSA[13, 16]. Target antifungal treatment was defined when appropriate target treatment started after susceptibility results, regardless of whether an inappropriate antifungal treatment has been initiated or not. Crude mortality was registered after 28 days from the occurrence of Candida BSI.

\section{Statistical analysis}

Descriptive analyses were used for baseline characteristics and subgroup analyses. Continuous variables were expressed as mean \pm standard deviation (SD). The Chi-square-test or 2-tailed Fisher Exact-test was applied to categorical variables. To define risk factors for mortality, multivariate Cox regression analysis, and adjusted hazard ratio (HR) with 95\% confidence interval (Cl) were calculated. Variables that were associated with 28-day mortality in Cox univariate analyses with a $\mathrm{P}<0.05$ were entered into multivariate cox analysis, based on the forward regression,2-tailed tests of significance at the level of a P value of $<0.05$ level was used to determine statistical significance. Statistical analysis has been performed with SPSS 22.0.

\section{Ethics}

The study was approved by the local institutional review board (Ruijin Hospital, Shanghai Jiaotong University, School of medicine) and written patient consent was not required because of the observational nature of this study.

\section{Results}

\section{Incidence and demographic characteristics of patients}

A total of 393 consecutive episodes of Candida BSI were collected, occurred among 370 inpatients, during an 11-year study period with an incidence of 0.39 episodes/1000 admissions, which has increased steadily, from 0.21 episodes per 1,000 admissions in 2008 to 0.59 in 2017 and 0.33 in 2018, as shown in Fig. 1. The demographic characteristics of the patients are summarized in Table 1. The mean age of patients was $57.6 \pm 19.0$ years and $74.3 \%$ were male. Among 393 episodes, 148 episodes (37.7\%) were from the surgical ward, 167 episodes (42.5\%) were from intensive care units (ICUs) and 78 episodes (19.8\%) were from the internal medicine ward, as shown in Fig. 2. 
Table 1

Demographic of patients with Candida bloodstream infection and distribution of Candida species

\begin{tabular}{|c|c|c|c|c|c|c|c|c|c|}
\hline & $\begin{array}{l}\text { C. } \\
\text { albicans }\end{array}$ & $\begin{array}{l}\text { C. } \\
\text { parapsilosis }\end{array}$ & $\begin{array}{l}\text { C. } \\
\text { tropicalis }\end{array}$ & $\begin{array}{l}\text { C. } \\
\text { glabrata }\end{array}$ & $\begin{array}{l}\text { C. } \\
\text { guilliermondii }\end{array}$ & C.sake & C.krusei & $\begin{array}{l}\text { Other } \\
\text { Candida } \\
\text { spp. }\end{array}$ & Total \\
\hline & $(n=141)$ & $(\mathrm{n}=87)$ & $(n=69)$ & $(n=48)$ & $(n=20)$ & $(n=8)$ & $(n=5)$ & $(n=15)$ & $(n=393)$ \\
\hline Age (year) & $\begin{array}{l}65.2 \pm \\
14.5\end{array}$ & $53.2 \pm 20.3$ & $\begin{array}{l}50.5 \pm \\
19.5\end{array}$ & $\begin{array}{l}60.7 \pm \\
17.5\end{array}$ & $50.5 \pm 19.4$ & $\begin{array}{l}52 \pm \\
20.6\end{array}$ & $\begin{array}{l}40.8 \pm \\
27.1\end{array}$ & $\begin{array}{l}52.6 \pm \\
20.0\end{array}$ & $\begin{array}{l}57.6 \pm \\
19.0\end{array}$ \\
\hline Male [n (\%)] & $102(73.4)$ & $60(69.0)$ & $51(73.9)$ & $44(91.7)$ & $14(70)$ & $8(100)$ & $2(40)$ & $11(73.3)$ & $292(74.3)$ \\
\hline \multicolumn{10}{|l|}{ Origin [n (\%)] } \\
\hline $\begin{array}{l}\text { Internal medicine } \\
\text { ward }\end{array}$ & 15(10.6) & 19(21.8) & $27(39.1)$ & $7(14.6)$ & $3(15)$ & $0(0)$ & $3(60)$ & $4(26.7)$ & 78(19.8) \\
\hline Surgical ward & $61(43.3)$ & $32(36.8)$ & $16(23.2)$ & 15(31.3) & $10(50)$ & $6(75)$ & $0(0)$ & $8(53.3)$ & 148(37.7) \\
\hline ICU & $65(46.1)$ & $36(41.4)$ & $26(37.7)$ & $26(54.1)$ & $7(35)$ & $2(25)$ & $2(40)$ & $3(20)$ & $167(42.5)$ \\
\hline $\begin{array}{l}\text { Time from } \\
\text { admission to } \\
\text { infection (d) }\end{array}$ & $\begin{array}{l}30.6 \pm \\
35.3\end{array}$ & $48.4 \pm 56.2$ & $\begin{array}{l}37.7 \pm \\
32.4\end{array}$ & $\begin{array}{l}27.5 \pm \\
19.1\end{array}$ & $35.9 \pm 52.5$ & $\begin{array}{l}120.6 \\
\pm \\
242.7\end{array}$ & $\begin{array}{l}64.2 \pm \\
68.6\end{array}$ & $\begin{array}{l}21.5 \pm \\
13.6\end{array}$ & $\begin{array}{l}37.6 \pm \\
53.1\end{array}$ \\
\hline $\begin{array}{l}\text { Length of hospital } \\
\text { stay (d) }\end{array}$ & $\begin{array}{l}56.0 \pm \\
54.9\end{array}$ & $83.2 \pm 78.1$ & $\begin{array}{l}71.5 \pm \\
56.1\end{array}$ & $\begin{array}{l}67.1 \pm \\
72.7\end{array}$ & $97.7 \pm 142.6$ & $\begin{array}{l}178.5 \\
\pm \\
281.7\end{array}$ & $\begin{array}{l}92.4 \pm \\
88.5\end{array}$ & $\begin{array}{l}52.7 \pm \\
101.5\end{array}$ & $\begin{array}{l}71.1 \pm \\
82.8\end{array}$ \\
\hline Turnaround Time (d) & $4.3 \pm 1.9$ & $4.5 \pm 1.1$ & $3.8 \pm 1.1$ & $4.8 \pm 1.3$ & $4.3 \pm 1.2$ & $\begin{array}{l}4.5 \pm \\
1.6\end{array}$ & $\begin{array}{l}4.4 \pm \\
1.5\end{array}$ & $5.9 \pm 1.8$ & $4.4 \pm 1.5$ \\
\hline \multicolumn{10}{|c|}{$\begin{array}{l}\text { Other Candida spp. Includes } C \text {. gum ( } 4 \text { cases), C. lusitaniae ( } 3 \text { cases), C. intermedia ( } 2 \text { cases), C. lipolytica (2cases), C. theae ( } 2 \text { cases), } \\
\text { C.famata ( } 1 \text { case) and C. haemulonii ( } 1 \text { case). }\end{array}$} \\
\hline
\end{tabular}

Table 1 here

C. albicans was isolated in $19.3 \%$ of cases in internal medicine wards while in $41.2 \%$ and $38.9 \%$ of cases in surgery wards and ICU, respectively $(\mathrm{P}=0.003)$. In contrast, a higher proportion of $C$. tropicalis $(34.7 \%)$ was found in internal medicine wards compared to that in surgery wards $(21.6 \%)$ and ICUs (21.6\%).

\section{Underlying Diseases And Clinical Features}

The majority of patients with Candida BSI had at least one co-morbidity. 118 (30\%) patients had solid tumors, 48 (12.2\%) patients had hematological malignancies, 77 (19.6\%) patients had diabetes mellitus, 124 (31.6\%) patients had chronic cardiac disease, 52 (13.2\%) patients had chronic pulmonary disease, 42 (10.7\%) patients had chronic renal failure, 26 (6.6\%) patients' skin barrier had been considered compromised, 244 (62.1\%) patients had prior surgical intervention, 54 (13.7\%) patients with corticosteroid use, 88 (22.4\%) patients had prior antifungal agents use, 255 (64.9\%) patients received antibiotics prior Candida BSI onset. 244 (72\%) patients had at least two co-morbidities. No patient had human immunodeficiency virus (HIV) infection. Regarding the severity, 309 (78.6\%) patients had fever, 180 (45.8\%) patients received parenteral nutrition, 147 (37.4\%) patients received mechanical ventilation, 49 (12.5\%) patients received renal replacement therapy, and $42(10.7 \%)$ patients had neutropenia. The clinical characteristics of patients, by Candida species, are shown in Table 2. 
Table 2

Underlying disease and clinical feature of Candida blood stream infection [n (\%)]

\begin{tabular}{|c|c|c|c|c|c|c|c|c|c|}
\hline & $\begin{array}{l}\text { C. } \\
\text { albicans }\end{array}$ & $\begin{array}{l}\text { C. } \\
\text { parapsilosis }\end{array}$ & $\begin{array}{l}\text { C. } \\
\text { tropicalis }\end{array}$ & $\begin{array}{l}\text { C. } \\
\text { glabrata }\end{array}$ & $\begin{array}{l}\text { C. } \\
\text { guilliermondii }\end{array}$ & C. sake & $\begin{array}{l}\text { C. } \\
\text { krusei }\end{array}$ & $\begin{array}{l}\text { Other } \\
\text { Candida } \\
\text { spp. }\end{array}$ & Total \\
\hline & $(n=141)$ & $(n=87)$ & $(n=69)$ & $(n=48)$ & $(n=20)$ & $(n=8)$ & $\begin{array}{l}(n= \\
5)\end{array}$ & $(n=15)$ & $(n=393)$ \\
\hline \multicolumn{10}{|l|}{ Underlying disease } \\
\hline Solid tumor & 47(33.3) & 26(29.9) & 13(18.8) & $17(35.4)$ & $6(30)$ & $2(25)$ & $0(0)$ & $7(46.7)$ & 118(30) \\
\hline $\begin{array}{l}\text { Hematologic } \\
\text { malignancy }\end{array}$ & $8(5.7)$ & $6(6.9)$ & $24(34.8)$ & $2(4.2)$ & $2(10)$ & $1(12.5)$ & $3(60)$ & 2(13.3) & $48(12.2)$ \\
\hline Diabetes mellitus & $33(23.4)$ & $21(24.1)$ & $8(11.6)$ & $9(18.8)$ & $1(5)$ & $1(12.5)$ & $0(0)$ & $4(26.7)$ & $77(19.6)$ \\
\hline $\begin{array}{l}\text { Chronic cardiac } \\
\text { disease }\end{array}$ & 55(39) & $22(25.3)$ & 16(23.2) & 18(37.5) & $6(30)$ & $3(37.5)$ & $2(40)$ & $2(13.3)$ & 124(31.6) \\
\hline $\begin{array}{l}\text { Chronic pulmonary } \\
\text { disease }\end{array}$ & $26(18.4)$ & $9(10.3)$ & $5(7.2)$ & $6(12.5)$ & $1(5)$ & $1(12.5)$ & $1(20)$ & $3(20)$ & $52(13.2)$ \\
\hline Chronic renal failure & $18(12.8)$ & $6(6.9)$ & $6(8.7)$ & $5(10.4)$ & $5(25)$ & $0(0)$ & $0(0)$ & 2(13.3) & 42(10.7) \\
\hline $\begin{array}{l}\text { Skin barrier } \\
\text { compromised }\end{array}$ & $5(3.5)$ & $9(10.3)$ & $5(7.2)$ & $1(2.1)$ & $3(15)$ & $3(37.5)$ & $0(0)$ & $0(0)$ & $26(6.6)$ \\
\hline $\begin{array}{l}\text { Prior surgical } \\
\text { intervention }(<1 \\
\text { month) }\end{array}$ & $97(68.8)$ & $48(55.2)$ & $36(52.2)$ & $30(62.5)$ & $15(75)$ & $6(75)$ & $2(40)$ & $10(66.7)$ & $244(62.1)$ \\
\hline Corticosteroid use & $11(7.8)$ & $13(14.9)$ & 12(17.4) & $9(18.8)$ & $4(20)$ & $0(0)$ & $3(60)$ & $2(13.3)$ & $54(13.7)$ \\
\hline $\begin{array}{l}\text { Prior use of antifungal } \\
\text { agents ( }<6 \text { months) }\end{array}$ & $20(14.2)$ & 19(21.8) & $24(34.8)$ & $11(22.9)$ & $8(40)$ & $1(12.5)$ & $4(80)$ & $1(6.7)$ & $88(22.4)$ \\
\hline \multicolumn{10}{|c|}{ Severity of clinical feature } \\
\hline Fever $\left(\mathrm{T}>38.2^{\circ} \mathrm{C}\right)$ & $114(80.9)$ & $63(72.4)$ & $60(87)$ & $34(70.8)$ & 16(80) & $6(75)$ & $3(60)$ & 13(86.7) & $309(78.6)$ \\
\hline Parenteral nutrition & $71(50.4)$ & $42(48.3)$ & $28(40.6)$ & $23(47.9)$ & $5(25)$ & $3(37.5)$ & $2(40)$ & $6(40)$ & $180(45.8)$ \\
\hline Mechanical ventilation & $58(41.1)$ & $31(35.6)$ & 23(33.3) & $25(52.1)$ & $5(25)$ & $2(25)$ & $2(40)$ & $1(6.7)$ & $147(37.4)$ \\
\hline $\begin{array}{l}\text { Renal replacement } \\
\text { therapy }\end{array}$ & $17(12.1)$ & $10(11.5)$ & $9(13)$ & $7(14.6)$ & $5(25)$ & $0(0)$ & $1(20)$ & $0(0)$ & $49(12.5)$ \\
\hline $\begin{array}{l}\text { Central venous } \\
\text { catheter }\end{array}$ & $121(85.8)$ & $67(77)$ & $49(71)$ & $43(89.6)$ & $17(85)$ & $5(62.5)$ & $4(80)$ & $10(66.7)$ & $316(80.4)$ \\
\hline Neutropenia & $4(2.8)$ & $8(9.2)$ & 22(31.9) & $1(2.1)$ & $2(10)$ & $0(0)$ & $3(60)$ & $2(13.3)$ & $42(10.7)$ \\
\hline 28-day mortality & $54(38.3)$ & $16(18.4)$ & $19(27.5)$ & $13(27.1)$ & $3(15)$ & $1(12.5)$ & $1(20)$ & $5(33.3)$ & $112(28.5)$ \\
\hline
\end{tabular}

Table 2 here

\section{Candida species and antifungal susceptibility testing}

393 Candida species were isolated in total. 141 (35.9\%) of Candida BSI were due to C. albicans, followed by C. parapsilosis(87cases, 22.1\%), C. tropicalis(69cases, 17.6\%), C. glabrata(48cases, 12.2\%), C. guilliermondii(20cases, 5.1\%), C. sake(8cases, 2.0\%), C.krusei(5cases, $1.3 \%)$, and 15 other species (4 C. gum, 3 C. Iusitaniae, 2 C. intermedia, 2 C. theae, 2 C. lipolytica, 1 C. famataand 1 C. haemulonii).

Among 393 Candida species, there were 378 episodes with antifungal susceptibility testing results. According to CLSI breakpoints 2012 (CBPs). As shown in Table 3, the susceptibility of $C$. albicans, $C$. parapsilosis to fluconazole and voriconazole were quite high, compared to itraconazole $(94 \%, 93.3 \%$ VS $82.1 \%)$. The susceptibility of $C$. tropicalis to triazoles fluconazole, voriconazole, or itraconazole was not satisfactory. Amphotericin B and 5-flucytosine remained superior to 95\% susceptibility against common Candida spp., except for $C$. krusei and C. guilliermondii. Because echinocandin susceptibility testing has not been carried out in our hospital, the relevant clinical data could not be obtained. 
Table 3

Antifungal susceptibility testing results (ATB Fungus 3) of 378 Candida [n (\%)]

\begin{tabular}{|c|c|c|c|c|c|c|c|c|c|}
\hline & $\begin{array}{l}\text { C.albicans } \\
(n=134)\end{array}$ & $\begin{array}{l}\text { C.parapsilosis } \\
(\mathrm{n}=86)\end{array}$ & $\begin{array}{l}\text { C.tropicalis } \\
(n=67)\end{array}$ & $\begin{array}{l}\text { C.glabrata } \\
(n=47)\end{array}$ & $\begin{array}{l}\text { C.krusei } \\
(\mathrm{n}=5)\end{array}$ & $\begin{array}{l}\text { C.sake } \\
(\mathrm{n}=8)\end{array}$ & $\begin{array}{l}\text { C.guilliermondii } \\
(n=19)\end{array}$ & $\begin{array}{l}\text { Other } \\
\text { Candida } \\
\text { spp. (n } \\
=12 \text { ) }\end{array}$ & $\begin{array}{l}\text { Total }(n= \\
378)\end{array}$ \\
\hline \multicolumn{10}{|c|}{ Fluconazole } \\
\hline$S$ & $126(94)$ & $77(89.5)$ & 35 (52.2) & $0(0)$ & $0(0)$ & $8(100)$ & $13(68.4)$ & $9(75.0)$ & $268(70.9)$ \\
\hline SDD & $1(0.8)$ & $6(7.0)$ & $3(4.5)$ & 44(93.6) & $0(0)$ & $0(0)$ & $0(0)$ & $0(0)$ & $54(14.3)$ \\
\hline $\mathrm{R}$ & $7(5.2)$ & $3(3.5)$ & $29(43.3)$ & $3(6.4)$ & $5(100)$ & $0(0)$ & $6(31.6)$ & $3(25.0)$ & $56(14.8)$ \\
\hline \multicolumn{10}{|c|}{ Itraconazole } \\
\hline$S$ & 110 (82.1) & 75 (87.2) & 25 (37.3) & $0(0)$ & $0(0)$ & $\begin{array}{l}8 \\
(100)\end{array}$ & 6 (31.6) & $9(75.0)$ & $233(61.6)$ \\
\hline SDD & $6(4.5)$ & $7(8.1)$ & $4(6.0)$ & $40(85.1)$ & $2(40.0)$ & $0(0)$ & $7(36.8)$ & $0(0)$ & $66(17.5)$ \\
\hline $\mathrm{R}$ & $18(13.4)$ & $4(4.7)$ & $38(56.7)$ & 7 (14.9) & $3(60.0)$ & $0(0)$ & $6(31.6)$ & $3(25.0)$ & $79(20.9)$ \\
\hline \multicolumn{10}{|c|}{ Voriconazole } \\
\hline$S$ & 125 (93.3) & 79 (91.9) & $41(61.2)$ & $45(95.8)$ & $4(80.0)$ & $\begin{array}{l}8 \\
(100)\end{array}$ & $12(63.2)$ & $\begin{array}{l}11 \\
(91.7)\end{array}$ & $325(86.0)$ \\
\hline SDD & $0(0)$ & $2(2.3)$ & $2(3.0)$ & $1(2.1)$ & $1(2.0)$ & $0(0)$ & $3(15.8)$ & $0(0)$ & $9(2.4)$ \\
\hline $\mathrm{R}$ & $9(6.7)$ & $5(5.8)$ & $24(35.8)$ & $1(2.1)$ & $0(0)$ & $0(0)$ & $4(21.0)$ & $1(8.3)$ & $44(11.6)$ \\
\hline \multicolumn{10}{|c|}{$\begin{array}{l}\text { Amphotericin } \\
\text { B }\end{array}$} \\
\hline S & 133 (99.3) & $83(96.5)$ & $67(100)$ & 47 (100) & $5(100)$ & $\begin{array}{l}8 \\
(100)\end{array}$ & $18(94.7)$ & $\begin{array}{l}11 \\
(91.7)\end{array}$ & $372(98.4)$ \\
\hline $\mathrm{R}$ & $1(0.7)$ & $3(3.5)$ & $0(0)$ & $0(0)$ & $0(0)$ & $0(0)$ & $1(5.3)$ & $1(8.3)$ & $6(1.6)$ \\
\hline \multicolumn{10}{|c|}{ Flucytosin } \\
\hline S & 132 (98.5) & 85 (98.8) & 65 (97.0) & $46(97.9)$ & $1(20.0)$ & $\begin{array}{l}8 \\
(100)\end{array}$ & $9(47.4)$ & $\begin{array}{l}12 \\
(100)\end{array}$ & $358(94.7)$ \\
\hline $\mathrm{R}$ & $2(1.5)$ & $1(1.2)$ & $2(3.0)$ & $1(2.1)$ & $4(80.0)$ & $0(0)$ & $10(52.6)$ & $0(0)$ & $20(5.3)$ \\
\hline \multicolumn{10}{|c|}{$\begin{array}{l}15 \text { Candida spp. isolats did not have susceptibility test, C. albicans (7), C. parapsilosis (2), C. tropicalis (2) and glabrata, theae, gum, } \\
\text { haemulonii each. }\end{array}$} \\
\hline
\end{tabular}

Table 3 here

\section{Antifungal therapy and outcome}

Antifungal therapy was administered in 299 (76.1\%) of the cases. 94 (23.9\%) patients did not receive any antifungal treatment. Among the patients who received antifungal therapy, 247 (62.8\%) received early appropriate antifungal therapy, and 52 (13.2\%) received target antifungal therapy. Fluconazole was most frequently used as empirical therapy, followed by echinocandins and voriconazole. 18 (4.6\%) patients with Candida BSI received combination therapy.

The overall, 28-day mortality rate was $28.5 \%$. The mortality rate was significantly higher in internal medicine wards and ICUs than in surgical wards $(37.2 \%$ and $34.7 \%$ vs. $16.9 \%$, respectively, $\mathrm{P}<0.001)$ (Fig. 3a). The mortality for those who received early appropriate or target antifungal therapy was $26.8 \%$ or $25.1 \%$ ( $P=0.012$ or $P=0.046)$, as compared to $39.3 \%$ for those who hadn't receive any antifungal therapy. However, there was no significant difference between the mortalities for those who received early appropriate antifungal therapy and for those who received target antifungal therapy (Fig. 3b).

In univariate analysis, age, solid tumor, diabetes mellitus, chronic cardiac disease, chronic renal failure, skin disease, prior surgical intervention, mechanical ventilation, neutropenia, and antifungal therapy were associated with 28-day mortality. In multivariate Cox regression analysis, advanced age $(H R=1.025 ; 95 \% C l, 1.013-1.037 ; \mathrm{P}<0.001)$, chronic renal failure $(\mathrm{HR}=2.018 ; 95 \% \mathrm{Cl} 1.234-3.299 ; \mathrm{P}=0.005)$, mechanical 
ventilation $(\mathrm{HR}=1.950 ; 95 \% \mathrm{Cl} 1.307-2.912 ; \mathrm{P}=0.001)$, neutropenia $(\mathrm{HR}=4.347 ; 95 \% \mathrm{Cl} 2.462-7.675 ; \mathrm{P}<0.001)$, were independent risk factors for 28-day mortality, while antifungal therapy $(\mathrm{HR}=0.570 ; 95 \% \mathrm{Cl} 0.382-0.849 ; \mathrm{P}=0.006)$ was independent protective factor for 28 -day mortality (Table 4).

Table 4

Multivariable Cox regression analysis for the risk factors about 28-day mortality of Candida bloodstream infection (393 episodes)

\begin{tabular}{|c|c|c|c|c|c|}
\hline \multirow[t]{2}{*}{ 28-day outcome } & \multirow[b]{2}{*}{ Survival $(n=281)$} & \multirow[b]{2}{*}{ Death $(n=112)$} & \multicolumn{3}{|c|}{ Multivariable analysis } \\
\hline & & & $P$ value & $\mathrm{HR}(95 \% \mathrm{Cl})$ & $P$ value \\
\hline Gender, male [n (\%)] & $216(76.9)$ & $76(67.9)$ & 0.065 & - & - \\
\hline Age (years, Mean \pm SD) & $55.2 \pm 19.5$ & $63.6 \pm 16.2$ & $<0.01$ & $1.025(1.013-1.037)$ & $<0.001$ \\
\hline Underlying disease & & & & - & - \\
\hline Solid tumor [n (\%)] & $91(32.4)$ & $27(24.1)$ & 0.106 & - & - \\
\hline Hematologic malignancy [n (\%)] & $32(11.4)$ & $16(14.3)$ & 0.428 & - & - \\
\hline Diabetes mellitus [n (\%)] & $49(17.4)$ & $28(25)$ & 0.088 & - & - \\
\hline Chronic Cardiac disease [n (\%)] & $72(25.6)$ & $52(46.4)$ & $<0.01$ & - & 0.105 \\
\hline Chronic Pulmonary disease [n (\%)] & $34(12.1)$ & $18(16.1)$ & 0.294 & - & - \\
\hline Chronic renal failure [n (\%)] & $20(7.1)$ & $22(19.6)$ & $<0.01$ & $2.018(1.234-3.299)$ & 0.005 \\
\hline Skin barrier compromised [n (\%)] & $24(8.5)$ & $2(1.8)$ & 0.015 & - & 0.308 \\
\hline Prior surgical intervention (<1month) [n (\%)] & $182(64.8)$ & $62(55.4)$ & 0.083 & - & - \\
\hline Corticosteroid use [n (\%)] & $40(14.2)$ & $14(12.5)$ & 0.652 & - & - \\
\hline Prior antifungal agents use (<6month) [n (\%)] & $64(22.8)$ & $24(21.4)$ & 0.772 & - & - \\
\hline Severity of clinical feature & & & & - & - \\
\hline Fever $\left(\mathrm{T}>38.2^{\circ} \mathrm{C}\right)[\mathrm{n}(\%)]$ & $220(78.3)$ & $89(79.5)$ & 0.798 & - & - \\
\hline Parenteral nutrition [n (\%)] & $124(44.1)$ & $56(50)$ & 0.292 & - & - \\
\hline Mechanical ventilation [n (\%)] & $89(31.7)$ & $58(51.8)$ & $<0.01$ & $1.950(1.307-2.912)$ & 0.001 \\
\hline Renal replacement therapy [n (\%)] & $32(11.4)$ & $17(15.2)$ & 0.305 & - & - \\
\hline Central venous catheter [n (\%)] & $227(80.8)$ & $89(79.5)$ & 0.766 & - & - \\
\hline Neutropenia [n (\%)] & $24(8.5)$ & $18(16.1)$ & 0.029 & $4.347(2.462-7.675)$ & $<0.001$ \\
\hline Antifungal therapy & $224(74.9)$ & $75(25.1)$ & 0.007 & $0.502(0.294-0.857)$ & 0.006 \\
\hline No treatment & $57(60.6)$ & $37(39.4)$ & & & \\
\hline
\end{tabular}

Table 4 here

\section{Discussion}

Our study showed that in our Tertiary-care hospital in Shanghai, the incidence of Candida BSI has increased steadily in the past 11 years. Several studies have shown a substantial increase in the past 2 decades, which was similar to our study[1, 8, 17]. Intensive use of broadspectrum antibiotics may be the main cause. Also, gradually worsening hospitalized patient profiles, underlying co-morbidities including malignancy, and high frequency of surgeries may be predisposing risk factors for increased incidence.

C. albicans was still the foremost pathogen of Candida BSI. However, over the past two decades, increased rates of common non-C. albicans Candida spp. has been reported worldwide, which was also observed in our study (35.9\% for C. albicans. and $64.1 \%$ for non-C. albicans). C. parapsilosis (34.5\%), C. tropicalis (27.4\%) and C. glabrata (19.0\%) account for most of non-C. albicans[18, 19]. In this study, the incidence of C. guilliermondii was incredibly higher than in other studies (4.8\% vs. 0.4\%)[20]. This could be related to the higher rate of parenteral nutrition and surgery (45.8\% and 62.1\%) in the current study, as some research implies that intravenous nutrition and surgery were significant risk factors for Candida BSI due to C. guilliermondii.[21]. 
The antifungal susceptibility testing showed that the susceptibility of C. albicans,

C. parapsilosis and C. sake for fluconazole were quite high (94\%, $89.5 \%, 100 \%)$, while the resistance rate of C. tropicalis for fluconazole was as high as $43.3 \%$, which was significantly higher than in other studies abroad[22, 23]. This may deserve more attention from clinicians in actual application.

There were $23.9 \%$ of patients with Candida BSI didn't receive any antifungal therapy in the present study. It was partly because some patients died before or soon after diagnostic confirmation. Although echinocandins have been recommended as a first-line antifungal agent, due to its high price, fluconazole was still the most frequently used empirical antifungal therapy. The 28-day crude mortality rate was $28.5 \%$; a statistically significant finding is that the 28-day mortality rate for Candida BSI in patients from surgical wards was quite low compared with other wards. The contributing factor needs further study.

Another finding in our study was that there was no significant difference in 28-day mortality rate between the patients who received early appropriate antifungal therapy and the patients who received target antifungal therapy ( $26.8 \%$ and $25.1 \%$ ). So we further analyzed demographic data, underlying diseases, and clinical features between these two groups, the result showed that there were no significant differences between the two groups in $\operatorname{age}(p=0.33)$, $\operatorname{sex}(p=0.89)$, the number of underlying diseases $(p=0.32)$ and the number of severe clinical features $(p=0.96)$. The choice of antifungal agents between these two groups was further analyzed. In the early appropriate antifungal therapy group, the rate of azoles use was $50.6 \%$, and the rate of echinocandin use was $30.3 \%$; while in the target antifungal therapy group, the rate of azoles use was $65.4 \%$, and the rate of echinocandin use was $17.3 \%$. The rate of echinocandin use was lower in the target antifungal therapy group than in the early appropriate antifungal therapy group $(p=0.038)$. Is the lower rate of echinocandin use in the target antifungal therapy group one of the reasons that caused no significant difference in mortality between the two groups? Many studies have confirmed the important role of echinocandin in antifungal therapy. Echinocandin has been recommended as a first-line antifungal agent since 2009[13], with fluconazole as an acceptable alternative for selected patients, reflecting the efficacy demonstrated by echinocandins and increasing resistance observed with fluconazole[24, 25]. Therefore, we believe that the lower rate of echinocandin use in the delayed antifungal treatment group is unlikely to lead to a decrease in the 28-day mortality rate. Based on the above analysis, we think that early antifungal therapy has no significant impact on the 28-day mortality rate compared with target antifungal therapy. Many clinicians currently administrate empirical antifungal agents; our study result may have an impact on their traditional view. Overuse use of antifungal drugs inevitably leads to a waste of medical resources and the rise of resistance[26]. If taking empirical antifungal agents cannot improve mortality, we should take a second thought about it. There are several studies support our findings[10-12], they showed that the severity of illness (APACHE-II Score) affected short-term survival in patients with Candida infection, whereas the choice of initial antifungal agents did not affect short-term survival. Trifi's research implied that no beneficial impact of an empirical antifungal therapy on 28- day survival neither in preventing the occurrence of candidemia in non-neutropenic septic critically patients[27]. However, some studies had different opinions on the timing of antifungal agents' use. Bassetti's research showed that the use of antifungal agents within 48 hours of obtaining a positive Candida blood culture result is an independent protective factor for mortality during hospitalization[9]; Similarly, Tedeschi's research showed that the administration of appropriate antifungal agents within 72 hours after blood culture was a protective factor for mortality during hospitalization[28]. However, due to the single-centered and the retrospective natures of their studies, those results need to be confirmed by more studies. Despite that, our results have shown similar risk factors (for example, age, neutropenia, mechanical ventilation, etc.) and protective factors (antifungal therapy) for 28-day mortality to theirs.

Although our data have been collected from a large hospital in Shanghai, several limitations of this study should be taken into consideration. First and foremost, this was a retrospective study performed at a single center, which could lead to selection bias. Moreover, the severity of illness score (such as APACHE II score), the timing of CVC removal was not included because of missing data.

\section{Conclusion}

Our retrospective study showed that the incidence of Candida BSI has increased in the past 11 years in Shanghai. Although the percentage of non- C. albicans has been increasing, the C. albicans remained the most frequently isolated species. The mortality of patients with Candida bloodstream infection was quite high, especially for those in internal medicine wards. Antifungal therapy did improve the short-term survival of patients with Candida BSI. Whether we should initiate preemptive antifungal therapy or apply antifungal therapy after antifungal susceptibility test needs further discussion.

\section{Abbreviations}

BSI

bloodstream infections

SD 
standard deviation

HR

hazard ratio

$\mathrm{Cl}$

confidence interval

ICU

intensive care units

HIV

human immunodeficiency virus

\section{Declarations}

\section{Ethics approval and consent to participate:}

The study was approved by the local institutional review board (Ruijin Hospital, Shanghai Jiaotong University, School of medicine) and written patient consent was not required because of the observational nature of this study.

\section{Consent for publication:}

Not applicable.

\section{Availability of data and materials:}

All data generated or analyzed during this study are included in this published article.

\section{Competing interest:}

The authors declare that they have no competing interests.

\section{Funding:}

This work was supported by the Program for Outstanding Medical Academic Leader, National Nature Science Foundation of China [81772107,81772040], Scientific and Technological Innovation Act Program of Science and Technology Commission of Shanghai Municipality [18411950900], Key Discipline Construction Project of Shanghai Municipal Commission of Health and Family Planning [2016ZB0206], Clinical Research Innovation Project of Shanghai Hospital Development Center [SHDC12017116] and Program of Shanghai Jiao Tong University School of Medicine [DLY201803] to Er-Zhen Chen.

\section{Authors' contributions:}

ZTY, EZC, and EQM made substantial contributions to conception and design. ZTY, YJZ, LW, TX LZ, and XYL participated in the acquisition of data. ZTY, YJZ, and YC drafted the manuscript. ZTY and EZC revised it critically.

\section{Acknowledgments:}

We thank Ms. Wen-Jing ZHENG to produce the massive time first to the article English grammar, the usage, and so on has carried on the revision.

\section{References}

1. Bitar D, Lortholary O, Le Strat Y, Nicolau J, Coignard B, Tattevin P, Che D, Dromer F. Population-based analysis of invasive fungal infections, France, 2001-2010. Emerg Infect Dis. 2014;20(7):1149-55.

2. Jia X, Li C, Cao J, Wu X, Zhang L. Clinical characteristics and predictors of mortality in patients with candidemia: a six-year retrospective study. European Journal of Clinical Microbiology Infectious Diseases. 2018;37(9):1717-24.

3. Marchetti O, Bille J, Fluckiger U, Eggimann P, Ruef C, Garbino J, Calandra T, Glauser M-P, Täuber MG, Pittet D, et al. Epidemiology of Candidemia in Swiss Tertiary Care Hospitals: Secular Trends, 1991-2000. Clin Infect Dis. 2004;38(3):311-20.

4. Wisplinghoff H, Bischoff T, Tallent SM, Seifert H, Wenzel RP, Edmond MB. Nosocomial Bloodstream Infections in US Hospitals: Analysis of 24,179 Cases from a Prospective Nationwide Surveillance Study. Clin Infect Dis. 2004;39(3):309-17.

5. Horn DL, Neofytos D, Anaissie EJ, Fishman JA, Steinbach WJ, Olyaei AJ, Marr KA, Pfaller MA, Chang CH, Webster KM. Epidemiology and outcomes of candidemia in 2019 patients: data from the prospective antifungal therapy alliance registry. Clinical infectious diseases: an 
official publication of the Infectious Diseases Society of America. 2009;48(12):1695-703.

6. Wisplinghoff H, Ebbers J, Geurtz L, Stefanik D, Major Y, Edmond MB, Wenzel RP, Seifert H. Nosocomial bloodstream infections due to Candida spp. in the USA: species distribution, clinical features and antifungal susceptibilities. Int J Antimicrob Agents. 2014;43(1):78-81.

7. Kim SH, Yoon YK, Kim MJ, Sohn JW. Clinical impact of time to positivity for Candida species on mortality in patients with candidaemia. J Antimicrob Chemother. 2013;68(12):2890-7.

8. Tiraboschi IN, Pozzi NC, Farias L, Garcia S, Fernandez NB: [Epidemiology, species, antifungal resistance and outcome of candidemia in a university hospital in Buenos Aires, Argentina for 16 years]. Revista chilena de infectologia: organo oficial de la Sociedad Chilena de Infectologia 2017, 34(5):431-440.

9. Bassetti M, Molinari MP, Mussap M, Viscoli C, Righi E. Candidaemia in internal medicine departments: the burden of a rising problem. Clin Microbiol Infect. 2013;19(6):E281-4.

10. Parkins MD, Sabuda DM, Elsayed S, Laupland KB. Adequacy of empirical antifungal therapy and effect on outcome among patients with invasive Candida species infections. J Antimicrob Chemother. 2007;60(3):613-8.

11. Taur Y, Cohen N, Dubnow S, Paskovaty A, Seo SK. Effect of antifungal therapy timing on mortality in cancer patients with candidemia. Antimicrob Agents Chemother. 2010;54(1):184-90.

12. Grim SA, Berger K, Teng C, Gupta S, Layden JE, Janda WM, Clark NM. Timing of susceptibility-based antifungal drug administration in patients with Candida bloodstream infection: correlation with outcomes. J Antimicrob Chemother. 2011;67(3):707-14.

13. Pappas PG, Kauffman CA, Andes D, Benjamin DK Jr, Calandra TF, Edwards JE Jr, Filler SG, Fisher JF, Kullberg B-J, Zeichner LO, et al. Clinical Practice Guidelines for the Management Candidiasis: 2009 Update by the Infectious Diseases Society of America. Clin Infect Dis. 2009;48(5):503-35.

14. Li F, Wu L, Cao B, Zhang Y, Li X, Liu Y. Surveillance of the prevalence, antibiotic susceptibility, and genotypic characterization of invasive candidiasis in a teaching hospital in China between 2006 to 2011. BMC Infect Dis. 2013;13:353.

15. Zhang L, Wang H, Xiao M, Kudinha T, Mao LL, Zhao HR, Kong F, Xu YC. The widely used ATB FUNGUS 3 automated readings in China and its misleading high MICs of Candida spp. to azoles: challenges for developing countries' clinical microbiology labs. PloS one. 2014;9(12):e114004.

16. Pappas PG, Kauffman CA, Andes DR, Clancy CJ, Marr KA, Ostrosky-Zeichner L, Reboli AC, Schuster MG, Vazquez JA, Walsh TJ, et al: Clinical Practice Guideline for the Management of Candidiasis: 2016 Update by the Infectious Diseases Society of America. Clinical infectious diseases: an official publication of the Infectious Diseases Society of America 2016, 62(4):e1-50.

17. Ulu Kilic A, Alp E, Cevahir F, Ture Z, Yozgat N: Epidemiology and cost implications of candidemia, a 6-year analysis from a developing country. Mycoses 2017, 60(3):198-203.

18. Chapman B, Slavin M, Marriott D, Halliday C, Kidd S, Arthur I, Bak N, Heath CH, Kennedy K, Morrissey CO, et al. Changing epidemiology of candidaemia in Australia. J Antimicrob Chemother. 2016;72(4):1103-8.

19. Sadeghi G, Ebrahimi-Rad M, Mousavi SF, Shams-Ghahfarokhi M, Razzaghi-Abyaneh M. Emergence of non-Candida albicans species: Epidemiology, phylogeny and fluconazole susceptibility profile. Journal de Mycologie Médicale. 2018;28(1):51-8.

20. Pfaller MA, Andes DR, Diekema DJ, Horn DL, Reboli AC, Rotstein C, Franks B, Azie NE. Epidemiology and outcomes of invasive candidiasis due to non-albicans species of Candida in 2,496 patients: data from the Prospective Antifungal Therapy (PATH) registry 2004-2008. PloS one. 2014;9(7):e101510.

21. Wu Z, Liu Y, Feng X, Liu Y, Wang S, Zhu X, Chen Q, Pan S. Candidemia: incidence rates, type of species, and risk factors at a tertiary care academic hospital in China. Int J Infect Dis. 2014;22:4-8.

22. Maria S, Barnwal G, Kumar A, Mohan K, Vinod V, Varghese A, Biswas R. Species distribution and antifungal susceptibility among clinical isolates of Candida parapsilosis complex from India. Revista Iberoamericana de Micología. 2018;35(3):147-50.

23. Neji S, Hadrich I, Trabelsi H, Abbes S, Cheikhrouhou F, Sellami H, Makni F, Ayadi A. Virulence factors, antifungal susceptibility and molecular mechanisms of azole resistance among Candida parapsilosis complex isolates recovered from clinical specimens. Journal of biomedical science. 2017;24(1):67.

24. Kett DH, Shorr AF, Reboli AC, Reisman AL, Biswas P, Schlamm HT. Anidulafungin compared with fluconazole in severely ill patients with candidemia and other forms of invasive candidiasis: support for the 2009 IDSA treatment guidelines for candidiasis. Crit Care (London England). 2011;15(5):R253.

25. Cui N, Wang H, Qiu H, Li R, Liu D. Impact of initial empirical antifungal agents on the outcome of critically ill patients with invasive candidiasis: analysis of the China-SCAN study. Int J Antimicrob Agents. 2017;50(1):74-80.

26. Hamdy RF, Zaoutis TE, Seo SK. Antifungal stewardship considerations for adults and pediatrics. Virulence. 2017;8(6):658-72. 
27. Trifi A, Abdellatif S, Daly F, Nasri R, Touil Y, Ben Lakhal S. Empiric antifungal and outcome in ICU patients. La Tunisie medicale. 2019;97(4):579-87.

28. Tedeschi S, Tumietto F, Giannella M, Bartoletti M, Cristini F, Cioni G, Ambretti S, Carretto E, Sambri V, Sarti M, et al. Epidemiology and outcome of candidemia in internal medicine wards: A regional study in Italy. Eur J Intern Med. 2016;34:39-44.

\section{Figures}

Figure 1.Distribution of Candida spp. and episodes/1000 admissions during study period (From 2008-2018)

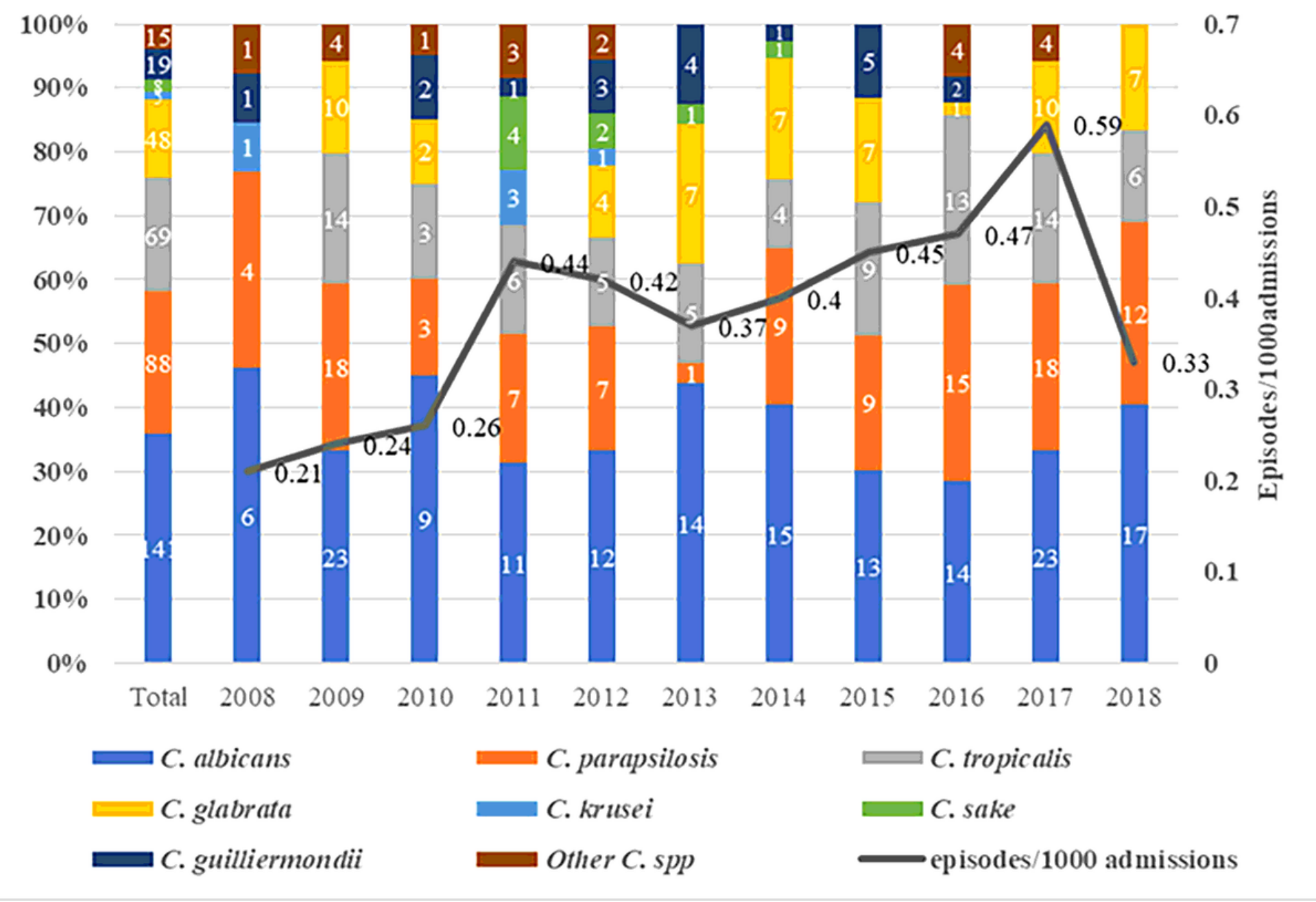

\section{Figure 1}

Distribution of Candida spp. and episodes/1000 admissions during study period (From 2008-2018). 
Figure 2. Distribution of Candida spp.

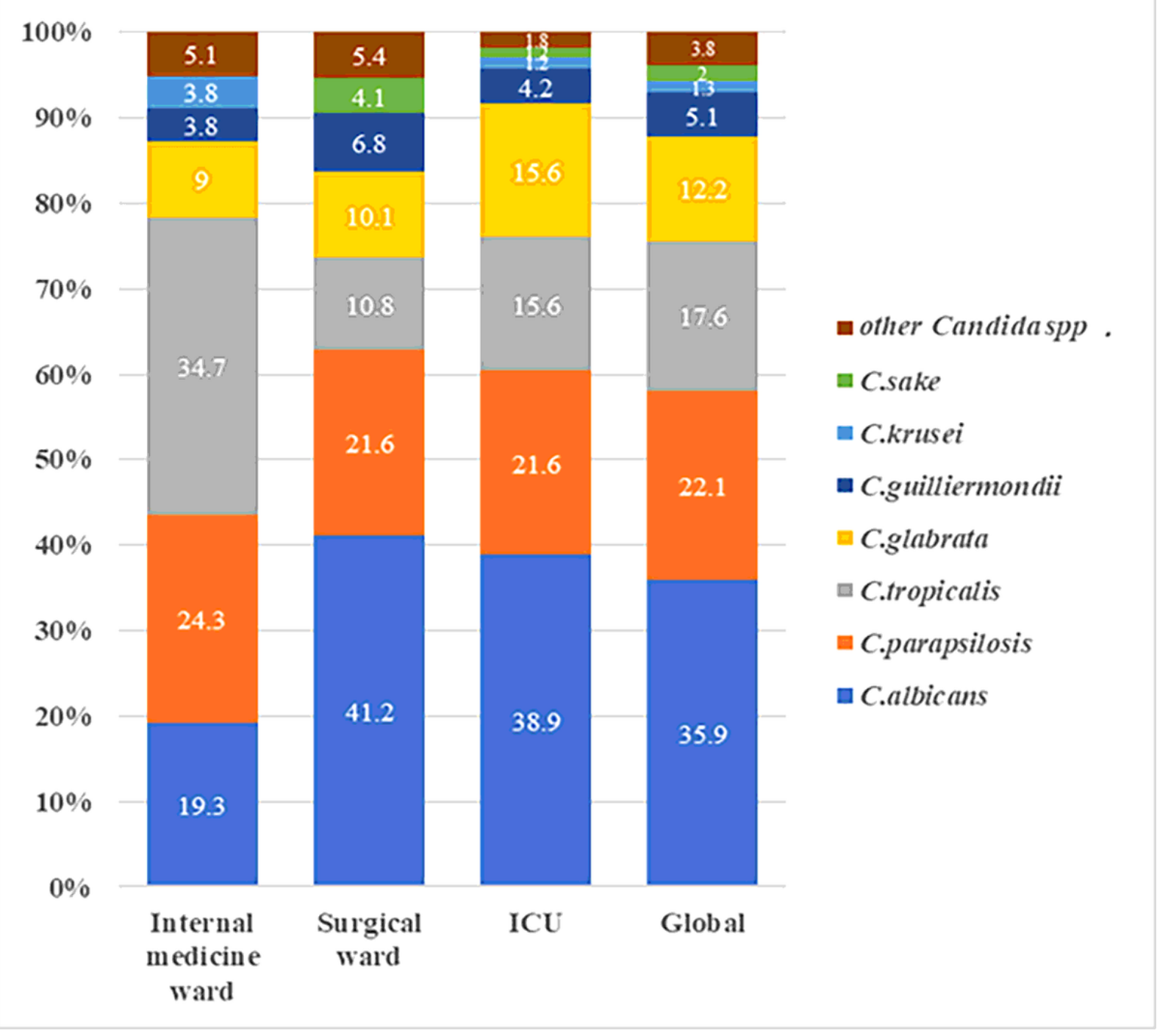

\section{Figure 2}

Distribution of Candida spp. 148 episodes were from the surgical ward, 167 episodes were from ICU and 78 episodes were from the internal medicine ward. 


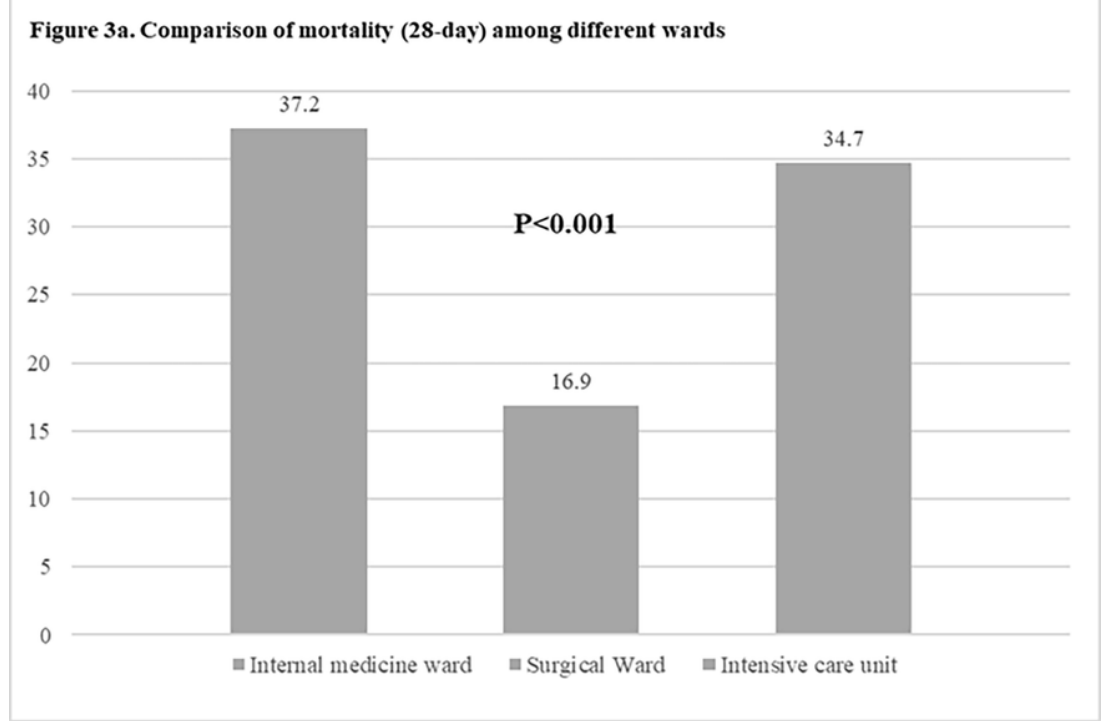

Figure 3b. Relationship between hospital mortality (28-day) and the timing of antifungal treatment.

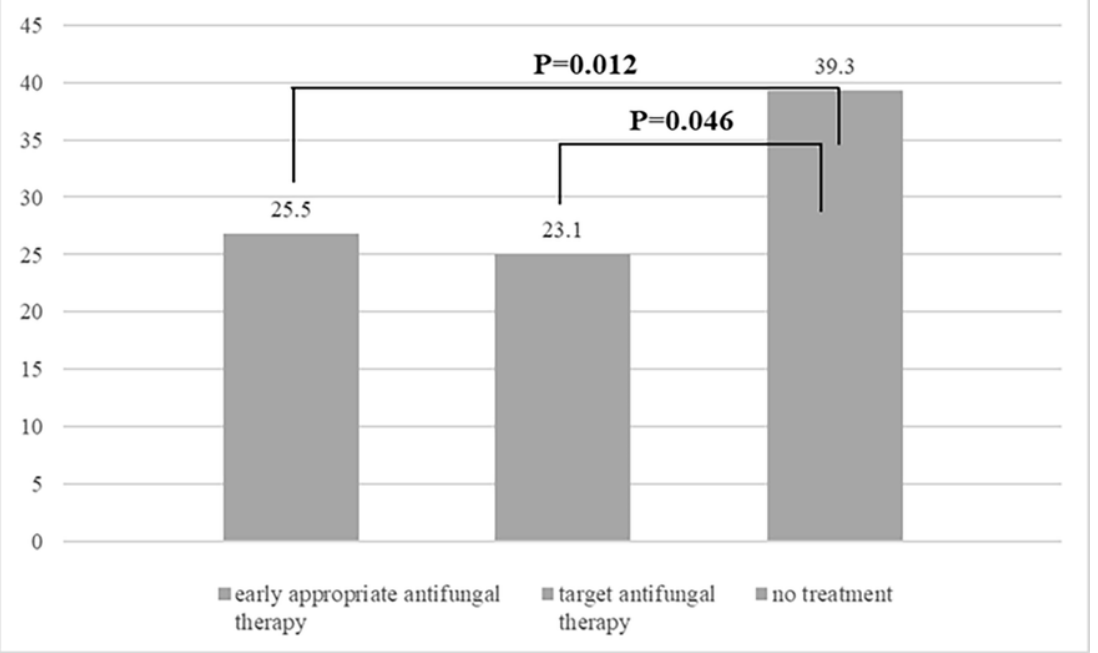

\section{Figure 3}

Figure 3a Comparison of mortality among different wards. The mortality rate was significantly higher in internal medicine wards and ICUs than in surgical wards Figure 3b Relationship between hospital mortality (28-day) and the timing of antifungal treatment. No significant difference between the mortalities for those who received early appropriate antifungal therapy and for those who received target antifungal therapy. 